\title{
Repeated Course Antenatal Steroids, Inflammation Gene Polymorphisms and Neurodevelopmental Outcomes at Age 2
}

\author{
Erin A. S. CLARK, M.D., Lisa MELE, Sc.M., Ronald J. WAPNER, M.D., Catherine Y. SPONG, \\ M.D., Yoram SOROKIN, M.D., Alan PEACEMAN, M.D., Jay D. IAMS, M.D., Kenneth J. \\ LEVENO, M.D., Margaret HARPER, M.D., Steve N. CARITIS, M.D., Brian M. MERCER, M.D., \\ John M. THORP, M.D., Susan M. RAMIN, M.D., Marshall CARPENTER, M.D., Dwight J. \\ ROUSE, M.D., and Eunice Kennedy Shriver National Institute of Child Health and Human \\ Development Maternal-Fetal Medicine Units Network ${ }^{*}$ \\ Departments of Obstetrics and Gynecology at the University of Utah, Salt Lake City, UT \\ (E.A.S.C.); Drexel University, Philadelphia, PA (R.J.W.); Wayne State University, Detroit, MI \\ (Y.S.); Northwestern University, Chicago, IL (A.P.); The Ohio State University, Columbus, OH \\ (J.D.I.); University of Texas Southwestern Medical Center, Dallas, TX (K.J.L.); Wake Forest \\ University Health Sciences, Winston-Salem, NC (M.H.); University of Pittsburgh, Pittsburgh, PA \\ (S.N.C.); Case Western Reserve University, Cleveland, OH (B.M.M.); University of North Carolina \\ at Chapel Hill, Chapel Hill, NC (J.M.T.); The University of Texas Health Science Center at \\ Houston, Houston, TX (S.M.R); Brown University, Providence, RI (M.C.); University of Alabama at \\ Birmingham, Birmingham, AL (D.J.R.); and The George Washington University Biostatistics \\ Center, Washington, DC (L.M.) and the Eunice Kennedy Shriver National Institute of Child Health \\ and Human Development, Bethesda, MD (C.Y.S.)
}

\section{Abstract}

OBJECTIVE-Evaluate the interaction between repeated course antenatal corticosteroids and inflammation gene polymorphisms with neurodevelopmental outcomes at age 2 .

\begin{abstract}
STUDY DESIGN—Nested case-control analysis of a randomized controlled trial of single versus repeated course antenatal corticosteroids. Cases had mental and/or psychomotor delay at age 2. Controls had normal neurodevelopment. Previous analyses of 125 cases and 147 controls identified 4 inflammation gene polymorphisms associated with neurodevelopmental delay at age 2.
\end{abstract}

\begin{abstract}
RESULTS-The interaction between repeated course corticosteroids and the IL6 -174 genotype with neurodevelopmental delay was significant $(\mathrm{P}=0.046)$. The IL6 -174 GG genotype was associated with neurodevelopmental delay at age 2 in the single course corticosteroid group (OR 6.47; 95\%CI 1.86-22.50). Exposure to repeated course antenatal corticosteroids abrogated this genotype effect (OR 1.30; 95\%CI 0.48-3.54). Results were unchanged after controlling for potential confounders.
\end{abstract}

\footnotetext{
(C) 2011 Mosby, Inc. All rights reserved.

Corresponding Author: Erin A. S. Clark, MD, University of Utah School of Medicine, Suite 2B200, 30 N 1900 E, Salt Lake City, UT 84132, business phone: 801-585-5156, fax: 801-585-2594, erin.clark@ @sc.utah.edu.

The other members of the National Institute of Child Health and Human Development (NICHD) Maternal-Fetal Medicine Units Network are listed in the Appendix.

Publisher's Disclaimer: This is a PDF file of an unedited manuscript that has been accepted for publication. As a service to our customers we are providing this early version of the manuscript. The manuscript will undergo copyediting, typesetting, and review of the resulting proof before it is published in its final citable form. Please note that during the production process errors may be discovered which could affect the content, and all legal disclaimers that apply to the journal pertain.

Presented at the 56th annual meeting of the Society of Gynecologic Investigation, Glasgow, Scotland, March 17-21, 2009
} 
CONCLUSION—Repeated course antenatal steroids may reduce the increased risk of neurodevelopmental delay at age 2 associated with IL6 -174 GG genotype.

\section{Keywords}

antenatal corticosteroids; interleukin-6 (IL6); inflammation; neurodevelopmental delay; gene polymorphisms

\section{INTRODUCTION}

Single course antenatal corticosteroid treatment is recommended for women at risk for preterm delivery, and is associated with a reduction in neonatal mortality and morbidity. ${ }^{1,2}$ The administration of repeated course antenatal corticosteroids has been the subject of recent clinical trials, and has been found to further reduce neonatal respiratory morbidity, relative to single-course regimens. ${ }^{3-5}$ However, these studies have also supported concerns regarding the impact of repeated course antenatal corticosteroids on fetal growth, head circumference and neurodevelopment. ${ }^{3,4,6,7}$

The clinical effects of antenatal corticosteroids may be mediated, in part, by their antiinflammatory effects. Corticosteroids affect inflammation signal transduction pathways by several different mechanisms: 1) increased synthesis of anti-inflammatory proteins, 2) inactivation of inflammatory genes, and 3) post-genomic effects leading to a reduction in inflammatory protein expression. ${ }^{8}$

The predominant effect of corticosteroids appears to be inhibition of inflammatory protein synthesis (e.g., pro-inflammatory cytokines) through gene suppression. Inflammatory stimuli result in activation of the transcription factor nuclear factor (NF)- $\kappa \beta$, which then translocates to the nucleus and binds to specific DNA recognition sequences, resulting in histone acetylation and increased expression of genes encoding pro-inflammatory cytokines (e.g., interleukin (IL)6 and IL1 $\beta$. There is persuasive evidence that corticosteroids inhibit the downstream effects of NF- $\kappa \beta$. The corticosteroid/glucocorticoid receptor complex translocates to the nucleus and binds to co-activators, leading to reversal of histone acetylation, decreased gene transcription, and decreased pro-inflammatory cytokine production. ${ }^{8}$

We have recently shown that single nucleotide polymorphisms (SNPs) in IL1 $\beta$ and IL6 genes may be associated with neurodevelopmental delay at age two years. ${ }^{9}$ ENREF 9 This is consistent with the reported associations between pro-inflammatory cytokines, perinatal white matter injury, and subsequent neurodevelopmental abnormalities. ${ }^{10}$ We hypothesized that the anti-inflammatory effects of repeated course antenatal corticosteroids would reduce the risk of neurodevelopmental delay at age 2 associated with pro-inflammatory gene SNPs.

\section{MATERIALS AND METHODS}

\section{Subjects}

Our subjects were the children of women enrolled in the Eunice Kennedy Shriver National Institute of Child Health and Human Development Maternal Fetal Medicine Units (MFMU) Network randomized, double-masked, placebo-controlled, multicenter clinical trial of single versus repeated courses of antenatal corticosteroids. The primary aim of the randomized trial was to assess the clinical efficacy and safety of repeated courses of antenatal corticosteroids in pregnancies at risk for preterm birth. All women in the trial received one course of either betamethasone or dexamethasone 6 to 10 days prior to enrollment. Consenting women were then randomly assigned to either weekly courses of betamethasone or placebo. The study 
protocol and results of the trial, which was conducted between 2000 and 2003, have been previously published. ${ }^{3}$ Placental samples and fetal cord serum were collected in a subset of subjects enrolled in the MFMU Network antenatal corticosteroid trial per study protocol.

A secondary aim of the MFMU Network antenatal corticosteroid trial was the correlation of steroid regimen with neurodevelopmental outcomes as assessed by the Bayley Scales of Infant Development at age 2 years. ${ }^{6}$ The Bayley Scales consist of mental and psychomotor developmental indices (MDI and PDI). A Bayley score of 85 is 1 SD below the mean and consistent with mild neurodevelopmental delay. A Bayley score of 70 is 2 SD below the mean and consistent with more significant delay. Overall, there were no significant differences between single versus repeated course treatment groups in Bayley scores. ${ }^{6}$

This secondary analysis aimed to evaluate whether there was an association between repeated course antenatal steroids and inflammation gene SNPs with neurodevelopmental outcomes at age 2 years. Inclusion criteria consisted of 1) Bayley scores at age 2 years and 2) available DNA for genotyping, either from fresh placental tissue, formalin-fixed paraffinimbedded placental tissue, or fetal cord serum collected at the time of delivery. Cases were children with mental and/or neurodevelopmental delay, defined by a Bayley MDI and/or PDI $<85$ at age 2 years. Cerebral palsy (CP) cases (as defined in the primary trial, $n=7$ ) ${ }^{3}$ were excluded from the analysis in order to test our hypothesis of an interaction between corticosteroid regimen and genotype on neurodevelopmental outcomes in the absence of CP. Controls were children with normal neurodevelopment, defined by Bayley MDI and PDI $\geq 85$.

Previously reported univariate analyses compared allele and genotype frequencies between cases and controls for 48 SNPs in inflammation and coagulation genes. ${ }^{9}$ ENREF 10 Significant genotype or allele differences were reported for IL1 $\beta$-511, IL6 -174 and -176, and IL4R 148. Of particular interest were the results for IL1 $\beta-511$ and IL6 -174, as these SNPs are in the promoter region of these genes and have been associated with altered gene expression and resultant functional levels of IL1 $\beta$ and IL6, respectively. ${ }^{11-15}$ The interaction between each of these inflammation gene SNPs and antenatal corticosteroid exposure with neurodevelopmental delay at age 2 was assessed in the current analysis.

The study protocol was reviewed by the Institutional Review Board (IRB) at the University of Utah and was determined to be exempt from IRB approval procedures secondary to deidentification of data and study samples prior to analysis. DNA extraction, genotyping, and quality control methods for this sample set have been previously reported. ${ }^{9}$

\section{Statistics}

Demographic and clinical characteristics of cases and controls were compared using Chisquare or Fisher's Exact test, to compare categorical variables, and the Wilcoxon Rank Sum test, to compare continuous variables. SNPs found to be significantly related to MDI and/or PDI $<85$ in prior univariate analyses $(\mathrm{p}<0.05)$ were further analyzed for interaction with antenatal corticosteroid exposure using the Breslow-Day test for homogeneity of the odds ratio and multivariable logistic regression modeling. The genotype model for each SNP was the best model determined in previous logistic regression analyses. ${ }^{9}$ Logistic regression model covariates included gestational age at delivery, gender, small for gestational age and steroid treatment group, as well as maternal race, smoking, and level of education. The sample size and the rarity of some genotypes and phenotypes limited further modeling of interactions. Tests for Hardy-Weinberg equilibrium (chi-square or Fisher's exact test) were performed on control subjects for each SNP significant in the univariate analysis. A nominal p-value of $<0.05$ was considered to be statistically significant. All calculations were performed using SAS (SAS Institute, Inc, Cary, NC). 


\section{RESULTS}

DNA from placental tissues and/or fetal serum was available for 272 infants, 125 cases and 147 controls. As previously described, ${ }^{9}$ most of the demographic and clinical characteristics of those included vs. excluded from the DNA analysis based on availability of biologic samples were similar. Study subjects with available samples tended to be older gestational age, greater birth weight, and from the single course treatment group. Cases and controls were similar in most characteristics, including gender, gestational age at delivery, chorioamnionitis at the time of delivery, maternal tobacco use, and exposure to repeated steroid courses. In cases and controls exposed to repeated steroid courses, the median number of study steroid courses after the initial course was 4 (range 1-8). Significant differences between cases and controls were observed for race/ethnicity and maternal education, covariates that were included in the logistic regression model.

Genotype determinations for IL6 -174 (rs1800795) were obtained for 135 subjects, 48 cases and 87 controls ( $\mathrm{G}$ allele wild-type, $\mathrm{C}$ allele minor). Of the 135 subjects, 63 patients were in the single course group and 72 were in the repeat course group. The Breslow-Day test for homogeneity of the odds ratio was significant $(\mathrm{P}=0.046)$ for IL6 -174, indicating the presence of an interaction between single versus repeated course antenatal corticosteroid exposure and genotype with risk of neurodevelopmental delay. The genotype distribution in children with neurodevelopmental delay versus controls, stratified by steroid regimen, is presented in Table 1.

Figure 1 shows the percentage of neurodevelopmental delay according to IL6 -174 genotype in the single versus repeated course steroid groups. In the single course steroid group, neurodevelopmental delay was significantly more common among infants with the GG genotype ( $54 \%$ versus $15 \%$ for the $\mathrm{GC} / \mathrm{CC}$ genotype, $\mathrm{p}=0.002$ ). In the repeated course steroid group, neurodevelopmental delay was not increased in infants with the GG genotype (36\% versus $30 \%$ for those with the GC/CC genotype, $\mathrm{p}=0.61$ ). Infants with the GG genotype had a higher rate of neurodevelopmental delay in the single course steroid group compared with the repeated course steroid group (54\% vs. $36 \%, \mathrm{P}=0.10)$. Conversely, the $\mathrm{GC} / \mathrm{CC}$ genotype group had a higher rate of neurodevelopmental delay in the repeated course corticosteroid group compared with the single course group ( $30 \%$ vs. $15 \%, \mathrm{P}=0.20$ ).

Examination by treatment group indicated that the IL6 -174 GG genotype was strongly associated with neurodevelopmental delay in the single course corticosteroid group (OR 6.47, 95\% CI of 1.86-22.50). Exposure to repeated course antenatal corticosteroids abrogated this genotype effect (OR 1.29, 95\%CI of 0.48-3.54). Results were unchanged after multivariable logistic regression analysis in the single course corticosteroid group (adjusted OR 6.56, 95\% CI 1.44-29.83) and in the repeated course corticosteroid group (adjusted OR $0.97,95 \%$ CI 0.27-3.53). (Table 2)

The remaining interactions between inflammation gene SNPs (IL1 $\beta$-511, IL6 -176, IL4R 148) and antenatal corticosteroid exposure with neurodevelopmental delay were not significant in this analysis (Table 1). In the control group, each SNP that was significant in the univariate analysis was in Hardy-Weinberg equilibrium, with the exception of IL6 -176 $(\mathrm{p}<0.001)$.

\section{COMMENT}

Our study suggests that the IL6 -174 GG genotype may be associated with neonatal developmental delay at age 2 , and that repeated courses of antenatal corticosteroids may reduce this genotype effect. We hypothesize, based on known corticosteroid-mediated antiinflammatory pathways, that the biologic mechanism may be through inactivation of the IL6 
gene, an increase in anti-inflammatory protein synthesis, and/or post-genomic reduction in IL6 protein expression.

Previous studies have demonstrated associations between pro-inflammatory cytokines, fetal/ neonatal brain injury and adverse long-term neurodevelopmental outcomes. Specifically, perinatal concentration of the pro-inflammatory cytokine IL6 has been correlated with the development of periventricular white matter injury and subsequent cerebral palsy. ${ }^{10}$ The fetal IL6 promoter genotype has been associated with adverse outcomes, including chorioamnionitis, preterm birth, and neonatal infection complications in multiple studies. ${ }^{16,17-19}$ In addition, variation in the IL6 gene promoter has been associated with impaired cognitive development in children born prematurely. ${ }^{20}$

The IL6 -174 SNP is in the enhancer-promoter region of the gene. Data regarding the function effect of this SNP on IL6 response have been conflicting. ${ }^{12-14,}$ 16, 21 The preponderance of evidence suggests that the $\mathrm{G}$ allele at position -174 is associated with a stronger IL6 response. It has also been associated with a higher incidence of histologic chorioamnionitis. ${ }^{12-14,16}$

Although we believe, based on biologic plausibility and our previous work, ${ }^{9}$ that neurodevelopmental delay associated with the GG genotype could be reduced with repeated exposure to antenatal corticosteroids through an anti-inflammatory mechanism, it is important to consider an alternative conclusion. The GC/CC genotype group had a higher rate of neurodevelopmental delay in the repeated course corticosteroid group compared with the single course group, although this difference was not significant. Although repeat courses of antenatal corticosteroids have not been associated with worse early neurocognitive and motor development relative to single-course regimens in randomized trials, it is possible that repeated steroid courses may have a detrimental effect on neurodevelopment in a genotypic subset of infants. Caution is therefore necessary in interpreting this gene-environment interaction, particularly given potential sample size limitations. Our findings are supported by our previous work which suggests that the GG genotype is associated with neurodevelopmental delay and by our a priori hypothesis that repeated course antenatal corticosteroids could reduce this effect through steroid-mediated antiinflammatory pathways. Further gene-environment analyses are necessary to support or refute our conclusions.

Although repeated courses of antenatal corticosteroids were associated with reduced risk of neurodevelopmental delay in infants with the IL6 -174 GG genotype, we do not propose that this is a viable therapeutic option for pregnancies at risk for preterm birth and subsequent adverse neurodevelopmental outcomes. Available evidence suggests that the risk-benefit ratio of administration of repeat courses of antenatal corticosteroids is unfavorable. Given concerns that repeat courses of antenatal steroids may negatively influence fetal growth, and since convincing evidence of long-term benefit is lacking, administration of repeated courses of antenatal steroids is not recommended. Rather, this study identifies corticosteroid and inflammation cell signaling pathways as potential targets for new neuroprotective strategies. Such neuroprotective strategies may be particularly important for fetuses with pro-inflammatory cytokine gene polymorphisms that may predispose to adverse neurodevelopmental outcomes.

SNPs in linkage disequilibrium with those we have identified, rather than the SNPs that we report, may be the actual causative variants associated with neurodevelopmental outcome. It should also be noted that neurodevelopmental testing at age 2 is of limited predictive value for long-term outcomes, particularly for subtle deficits. Whether the interaction of inflammation gene variants and exposure to antenatal corticosteroids has any clinically 
significant long-term neurocognitive effects is uncertain, and cannot be extrapolated from this data. In addition, the effect of inflammation gene polymorphisms on neurodevelopment in the absence of antenatal corticosteroid exposure cannot be evaluated in this analysis.

Sample size did not permit analysis with stratification by race/ethnicity or type of neurodevelopmental delay, and we acknowledge this as a study limitation. Although race/ ethnicity was a covariate included in logistic regression models, the genotype effect within race/ethnicity strata cannot be determined in this analysis. In addition, the relatively small number of confirmed cases of intrauterine infection/inflammation prevented correlation with neurodevelopmental outcomes, a gene-environment interaction of obvious interest. Although cerebral palsy is a neurodevelopmental outcome worthy of similar evaluation, these cases were uncommon and there would not have been adequate power to analyze this outcome.

The risk of central nervous system injury in preterm infants is influenced by complex geneenvironment interactions that are not yet well understood. This study offered a unique opportunity to evaluate a cohort of infants at risk for neurodevelopmental delay due to preterm birth, with well-characterized clinical outcomes, for a gene-environment interaction that may influence outcomes at age 2 years. This study adds to the current evidence that certain inflammation gene variants may contribute to adverse neurodevelopmental outcomes, and that this genetic predisposition may be modified by the antenatal environment.

\section{Acknowledgments}

The following subcommittee members participated in protocol development and coordination between clinical research centers (Michelle Di Vito, R.N. and Francee Johnson, R.N., B.S.N.), protocol/data management and statistical analysis (Elizabeth Thom, Ph.D.), and protocol development and oversight (Donald J. Dudley, M.D.). We also thank Dr. Michael Varner for his invaluable assistance with protocol development, manuscript preparation, and mentorship to Dr. Erin Clark.

Supported by grants from the Eunice Kennedy Shriver National Institute of Child Health and Human Development (HD21410, HD27869, HD27917, HD27860, HD27915, HD34116, HD34208, HD34136, HD40500, HD40485, HD40544, HD40545, HD40560, HD40512, HD36801) and M01-RR-000080 from the National Center for Research Resources

\section{APPENDIX}

In addition to the authors, other members of the Eunice Kennedy Shriver National Institute of Child Health and Human Development Maternal-Fetal Medicine Units Network are as follows:

University of Utah - D. Dudley, M. Varner, K. Anderson, A. Guzman, A. Crowley, M. Fuller

Drexel University - M. DiVito, A. Sciscione, V. Berghella, M. Pollock, M. Talucci

Wayne State University - M. Dombrowski, G. Norman, A. Millinder, C. Sudz, D. Driscoll

The Ohio State University - F. Johnson, M. Landon, S. Meadows, P. Shubert

Northwestern University — G. Mallett

University of Texas Southwestern Medical Center — D. Weightman, L. Fay-Randall, P. Mesa 
Wake Forest University Health Sciences - P. Meis, M. Swain, C. Moorefield

University of Pittsburgh - T. Kamon, K. Lain, M. Cotroneo

Columbia University - M. Miodovnik, F. Malone, V. Pemberton, S. Bousleiman

Case Western Reserve University - P. Catalano, C. Milluzzi, C. Santori

University of North Carolina at Chapel Hill - K. Moise, K. Dorman

The University of Texas Health Science Center at Houston — L. Gilstrap, M. Day

Brown University — D. Allard, J. Tillinghast

University of Alabama at Birmingham - A. Northern, K. Bailey

The George Washington University Biostatistics Center - E. Thom, F. Galbis-Reig, L. Leuchtenburg

Eunice Kennedy Shriver National Institute of Child Health and Human Development - D. McNellis, K. Howell, S. Pagliaro

MFMU Network Steering Committee Chair (Vanderbilt University Medical Center) - S. Gabbe

\section{REFERENCES}

1. Roberts D, Dalziel S. Antenatal corticosteroids for accelerating fetal lung maturation for women at risk of preterm birth. Cochrane Database Syst Rev. 2006; 3:CD004454. [PubMed: 16856047]

2. Crowley PA. Antenatal corticosteroid therapy: a meta-analysis of the randomized trials, 1972 to 1994. Am J Obstet Gynecol. 1995; 173:322-35. [PubMed: 7631713]

3. Wapner RJ, Sorokin Y, Thom EA, et al. Single versus weekly courses of antenatal corticosteroids: evaluation of safety and efficacy. Am J Obstet Gynecol. 2006; 195:633-42. [PubMed: 16846587]

4. Murphy KE, Hannah ME, Willan AR, et al. Multiple courses of antenatal corticosteroids for preterm birth (MACS): a randomised controlled trial. Lancet. 2008; 372:2143-51. [PubMed: 19101390]

5. Crowther CA, Haslam RR, Hiller JE, Doyle LW, Robinson JS. Neonatal respiratory distress syndrome after repeat exposure to antenatal corticosteroids: a randomised controlled trial. Lancet. 2006; 367:1913-9. [PubMed: 16765760]

6. Wapner RJ, Sorokin Y, Mele L, et al. Long-term outcomes after repeat doses of antenatal corticosteroids. N Engl J Med. 2007; 357:1190-8. [PubMed: 17881751]

7. Bevilacqua E, Brunelli R, Anceschi MM. Review and meta-analysis: Benefits and risks of multiple courses of antenatal corticosteroids. J Matern Fetal Neonatal Med. 2009:1-17.

8. Barnes PJ. Corticosteroid effects on cell signalling. Eur Respir J. 2006; 27:413-26. [PubMed: 16452600]

9. Clark EA, Mele L, Wapner RJ, et al. Association of fetal inflammation and coagulation pathway gene polymorphisms with neurodevelopmental delay at age 2 years. Am J Obstet Gynecol. 2010; 203:83, e1-e10. [PubMed: 20417488]

10. Yoon BH, Jun JK, Romero R, et al. Amniotic fluid inflammatory cytokines (interleukin-6, interleukin-1beta, and tumor necrosis factor-alpha), neonatal brain white matter lesions, and cerebral palsy. Am J Obstet Gynecol. 1997; 177:19-26. [PubMed: 9240577]

11. Chen H, Wilkins LM, Aziz N, et al. Single nucleotide polymorphisms in the human interleukin-1B gene affect transcription according to haplotype context. Hum Mol Genet. 2006; 15:519-29. [PubMed: 16399797] 
12. Fishman D, Faulds G, Jeffery R, et al. The effect of novel polymorphisms in the interleukin-6 (IL-6) gene on IL-6 transcription and plasma IL-6 levels, and an association with systemic-onset juvenile chronic arthritis. J Clin Invest. 1998; 102:1369-76. [PubMed: 9769329]

13. Castellucci L, Menezes E, Oliveira J, et al. IL6 -174 G/C promoter polymorphism influences susceptibility to mucosal but not localized cutaneous leishmaniasis in Brazil. J Infect Dis. 2006; 194:519-27. [PubMed: 16845637]

14. Rivera-Chavez FA, Peters-Hybki DL, Barber RC, O'Keefe GE. Interleukin-6 promoter haplotypes and interleukin-6 cytokine responses. Shock. 2003; 20:218-23. [PubMed: 12923492]

15. Rogus J, Beck JD, Offenbacher S, et al. IL1B gene promoter haplotype pairs predict clinical levels of interleukin-1beta and C-reactive protein. Hum Genet. 2008; 123:387-98. [PubMed: 18369665]

16. Reiman M, Kujari H, Ekholm E, Lapinleimu H, Lehtonen L, Haataja L. Interleukin-6 polymorphism is associated with chorioamnionitis and neonatal infections in preterm infants. $\mathrm{J}$ Pediatr. 2008; 153:19-24. [PubMed: 18571528]

17. Velez DR, Fortunato SJ, Williams SM, Menon R. Interleukin-6 (IL-6) and receptor (IL6-R) gene haplotypes associate with amniotic fluid protein concentrations in preterm birth. Hum Mol Genet. 2008; 17:1619-30. [PubMed: 18276608]

18. Simhan HN, Krohn MA, Roberts JM, Zeevi A, Caritis SN. Interleukin-6 promoter -174 polymorphism and spontaneous preterm birth. Am J Obstet Gynecol. 2003; 189:915-8. [PubMed: 14586325]

19. Hartel C, Finas D, Ahrens P, et al. Polymorphisms of genes involved in innate immunity: association with preterm delivery. Mol Hum Reprod. 2004; 10:911-5. [PubMed: 15516360]

20. Harding D, Brull D, Humphries SE, Whitelaw A, Montgomery H, Marlow N. Variation in the interleukin-6 gene is associated with impaired cognitive development in children born prematurely: a preliminary study. Pediatr Res. 2005; 58:117-20. [PubMed: 15985687]

21. Kilpinen S, Hulkkonen J, Wang XY, Hurme M. The promoter polymorphism of the interleukin-6 gene regulates interleukin-6 production in neonates but not in adults. Eur Cytokine Netw. 2001; 12:62-8. [PubMed: 11282548] 


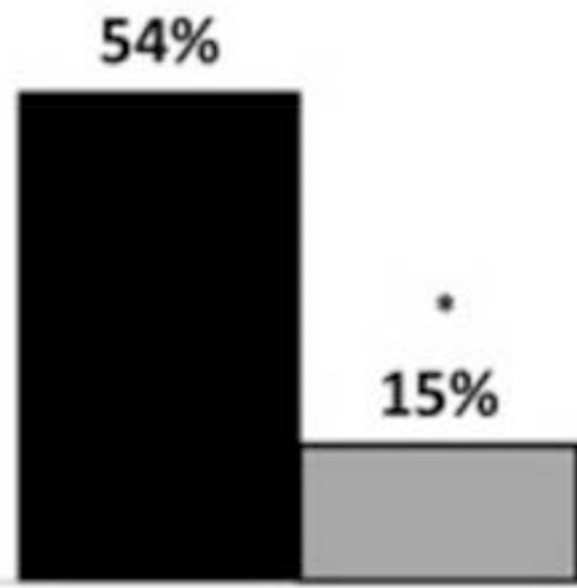

SINGLE COURSE STEROIDS

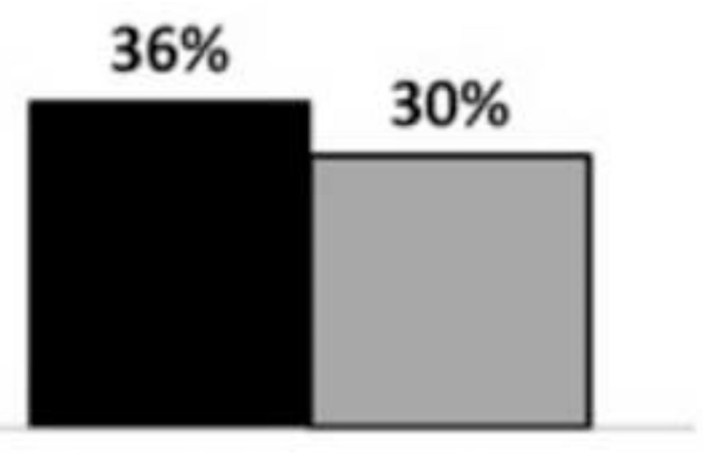

MULTIPLE COURSE STEROIDS

\section{- GG $\mathrm{GCC} / \mathrm{CC}$}

Figure 1.

In the single course steroid group, neurodevelopmental delay was significantly more common among infants with the GG genotype (54\% versus $15 \%$ for the GC/CC genotype, $\mathrm{p}=0.002)$. In the repeated course steroid group, neurodevelopmental delay was not increased in infants with the GG genotype (36\% versus $30 \%$ for those with the GC/CC genotype, $\mathrm{p}=0.61)$. 


\section{Table 1}

Genotype in Children with Neurodevelopmental Delay (Bayley MDI and/or PDI <85) versus Controls, Stratified by Steroid Regimen

\begin{tabular}{lllllll}
\hline & & \multicolumn{2}{l}{$\begin{array}{l}\text { Single Course } \\
\text { Corticosteroids }\end{array}$} & \multicolumn{2}{l}{$\begin{array}{l}\text { Repeated Course } \\
\text { Corticosteroids }\end{array}$} & P $^{*}$ \\
\hline SNP & Genotype & Cases & Controls & Cases & Controls & \\
\hline IL1 $\beta-511$ & AA/AG & $35(79.6)$ & $41(91.1)$ & $26(78.8)$ & $50(92.6)$ & 0.79 \\
\hline & GG & $9(20.5)$ & $4(8.9)$ & $7(21.2)$ & $4(7.4)$ & \\
\hline IL6 -174 & GG & $\mathbf{2 0}(\mathbf{8 3 . 3})$ & $\mathbf{1 7 ( 4 3 . 6 )}$ & $\mathbf{1 5}(\mathbf{6 2 . 5})$ & $\mathbf{2 7 ( 5 6 . 3 )}$ & $\mathbf{0 . 0 4 6}$ \\
\hline & GC/CC & $\mathbf{4 ( 1 6 . 7 )}$ & $\mathbf{2 2 ( 5 6 . 4 )}$ & $\mathbf{9 ( 3 7 . 5 )}$ & $\mathbf{2 1 ( 4 3 . 8 )}$ & \\
\hline IL6 -176 & GG & $21(35.6)$ & $32(51.6)$ & $19(46.3)$ & $37(57.8)$ & 0.72 \\
\hline & GC/CC & $38(64.4)$ & $30(48.4)$ & $22(53.7)$ & $27(42.2)$ & \\
\hline IL4R 148 & AA & $27(46.6)$ & $20(35.1)$ & $17(41.5)$ & $17(27.9)$ & 0.82 \\
\hline & AG/GG & $31(53.5)$ & $37(64.9)$ & $24(58.5)$ & $44(72.1)$ & \\
\hline
\end{tabular}

Data presented as $\mathrm{n}(\%)$. The genotype model for each SNP was the best model determined in previous logistic regression analyses. 9

Breslow Day Test for interaction 
Table 2

Association of Neurodevelopmental Delay with IL6 -174 GG Genotype in the Single versus Repeated Course Antenatal Corticosteroid Groups

\begin{tabular}{lcc}
\hline Antenatal Corticosteroid Treatment Group & OR $^{*}$ & $\mathbf{9 5 \%} \mathbf{C I}^{\dagger}$ \\
\hline Single Course & 6.56 & $1.44-29.83$ \\
Repeated Courses & 0.97 & $0.27-3.53$ \\
\hline
\end{tabular}

P-value for the interaction term in the logistic regression model was 0.044 , indicating the presence of an interaction between single versus repeated course antenatal corticosteroid exposure and IL6 -174 genotype with risk of neurodevelopmental delay.

Adjusted odds ratio adjusted for gestational age at delivery, gender, SGA, maternal race, smoking, and level of education.

$95 \%$ Confidence Interval 